\title{
Revising the Local Bubble Model due to Solar Wind Charge Exchange X-ray Emission
}

\author{
Robin L. Shelton
}

Received: date / Accepted: date

\begin{abstract}
The hot Local Bubble surrounding the solar neighborhood has been primarily studied through observations of its soft X-ray emission. The measurements were obtained by attributing all of the observed local soft X-rays to the bubble. However, mounting evidence shows that the heliosphere also produces diffuse X-rays. The source is solar wind ions that have received an electron from another atom. The presence of this alternate explanation for locally produced diffuse X-rays calls into question the existence and character of the Local Bubble. This article addresses these questions. It reviews the literature on solar wind charge exchange (SWCX) X-ray production, finding that SWCX accounts for roughly half of the observed local 1/4 keV X-rays found at low latitudes. This article also makes predictions for the heliospheric O VI column density and intensity, finding them to be smaller than the observational error bars. Evidence for the continued belief that the Local Bubble contains hot gas includes the remaining local $1 / 4 \mathrm{keV}$ intensity, the observed local $\mathrm{O}$ VI column density, and the need to fill the local region with some sort of plasma. If the true Local Bubble is half as bright as previously thought, then its electron density and thermal pressure are $1 / \sqrt{2}$ as great as previously thought, and its energy requirements and emission measure are $1 / 2$ as great as previously thought. These adjustments can be accommodated easily, and, in fact, bring the Local Bubble's pressure more in line with that of the adjacent material. Suggestions for future work are made.
\end{abstract}

Keywords Local Bubble: Interstellar Medium; Solar Wind Charge Exchange; Solar Wind; SWCX; Diffuse X-rays; O VII

\section{Introduction}

The Local Bubble (LB) is traditionally thought of as a large ( $\sim 60 \mathrm{pc}$ in radius) bubble filled with hot $\left(\sim 10^{6} \mathrm{~K}\right)$ plasma surrounding the Solar neighborhood. Several clouds of warm $\left(\sim 10^{4} \mathrm{~K}\right)$ gas reside within the Local Bubble. One of these clouds, the Local Cloud, envelops the Solar System.

The Local Bubble was discovered in the 1970's through 1/4 keV X-ray observations. Early X-ray instruments detected soft X-rays from every direction and with B and C band

Department of Physics and Astronomy, the University of Georgia, Athens, GA 30602 
(bandpasses: $\sim 70-188 \mathrm{eV}$ and $\sim 160-284 \mathrm{eV}$, respectively) intensities that anti-correlate with absorbing column density but correlate well with each other. These characteristics suggested that the Earth resides within an X-ray emissive bubble which has displaced neutral material in the disk (1). That some of the observed X-rays were produced locally and not, for example, in the Galactic halo or bulge, has been confirmed by shadowing studies in which absorbing interstellar clouds are used to block X-rays from more distant sources (2; 3). The $\mathrm{X}$-ray intensity observed in the direction of the cloud, minus the intensity of distant photons that have leaked through the cloud, indicates the brightness of the local region. Technically, these studies indicate only that X-rays were produced "locally", i.e. somewhere between the satellite and the absorbing cloud; these studies do not reveal the precise location of the Xray emitting gas. Although astronomers have long recognized the possibility that the Solar System contributed to the observed local X-ray intensity, the agreement between $1 / 4 \mathrm{keV}$ observations taken more than a year apart (4) required a constant emission source and so favored the interstellar interpretation. This changed in 1996 when ROSAT observed X-rays from the coma of Comet Hyakutake (5). These observations unequivocally demonstrated that the heliosphere contains X-ray sources aside from the Sun and therefore called into question the assumption that all of the locally produced diffuse X-ray flux was created by the Local Bubble. The existence and nature of the Local Bubble are now being reevaluated. This article addresses these issues. After further describing the historical Local Bubble model and diffuse Solar System X-ray emission, the paper proceeds to a discussion of whether or not the Solar System can entirely replace the Local Bubble as the explanation for the "local" emission (it cannot) and to a new picture of the Local Bubble which takes into account the presence of Solar System X-rays.

\section{The Historical Local Bubble Model}

As mentioned in the introduction, the historical Local Bubble's temperature is about a million degrees Kelvin. This temperature was found by comparing observed X-ray band ratios with models for optically thin hot gas in collisional ionizational equilibrium. Even the data from the earliest $1 / 4 \mathrm{keV} \mathrm{X}$-ray telescopes, in which the Local Bubble emission was not distinguished from that of the Galactic halo, matched that of a 0.9 to $1.2 \times 10^{6} \mathrm{~K}$ plasma (1). Later, ROSAT All Sky Survey data, for which it was possible to separate the Local Bubble and Galactic halo contributions, yielded a similar Local Bubble temperature $\left(T_{L B}=1.3 \times 10^{6} \mathrm{~K}\right.$, with variation from $T_{L B}=1.1$ to $1.9 \times 10^{6} \mathrm{~K}$ depending upon direction, (6)).

Warm clouds are buried deep within the bubble. These have been observed via their absorption of light from nearby stars and by analyses of the material that abuts the heliosphere (see other articles in this volume for further details). In addition to these temperature extremes, we expect to find intermediate temperature gas in the transition zones between the clouds and the hot bubble plasma. Thus, $\mathrm{O}$ VI, which traces $3 \times 10^{5} \mathrm{~K}$ gas was sought. It was found, both in a statistical analysis of dozens of Copernicus column density measurements (7) and, also in analyses of FUSE data for stars within about a hundred parsecs of the Sun $(8 ; 9)$. This distance range is thought to be similar to the Local Bubble's radius, which was determined from data on a molecular cloud residing just inside the bubble boundary (10). Once the radius was known for one direction, the radii for other directions were estimated from the observed intensities in those directions and the assumption that the temperature and density are the same in all directions. By this method, the distance from the Sun to the Local 
Bubble's periphery was found to vary from about $40 \mathrm{pc}$ to slightly more than $100 \mathrm{pc}(11)$. Measurements of $\mathrm{Na}$ I column densities toward nearby stars indicate that a larger cavity, called the Local Cavity, also surrounds the solar neighborhood. This region of exceptionally low volume density varies in radius from $\sim 40$ to $\sim 200 \mathrm{pc}(12)$. The Local Bubble fits easily within the Local Cavity.

Several models have been proposed to explain the Local Bubble, including a single supernova explosion $(13 ; 14)$, multiple supernova explosions $(15)$, and a bubble that has broken out of its nascent molecular cloud (16). Multiple supernova models are currently favored because of the large input energy requirement to blow such a large and energetic bubble and because the observed $1 / 4 \mathrm{keV}$ X-ray to O VI and C III ratios disallow the blowout model $(17 ; 18 ; 8)$.

It is noteworthy that time variability was seen in the ROSAT data. The ROSAT All Sky Survey scans overlapped each other. By comparing data taken during one orbit with that taken during the next, it was possible to see that the flux level varied with time in addition to position on the sky. The excess fluxes, called "long term enhancements" were nominally removed from the ROSAT All Sky Survey data before the data were used to measure the Local Bubble's temperature and brightness. The long term enhancements were unexplained and troubling. But, after X-rays were seen from Comet Hyakutake, (19) suggested that solar wind X-rays could have caused the long term enhancements in the ROSAT survey. A more troubling notion is the possibility that solar wind X-rays may cause additional contamination.

\section{Charge Exchange in the Solar System}

\subsection{Solar Wind Charge Exchange X-rays}

The physical mechanism by which Comet Hyakutake emitted X-rays also acts throughout the Solar System. In this mechanism, highly charged ions in the solar wind collide with and receive electrons from other atoms. The electrons transfer into high $n$ levels in the solar wind ions, then they radiatively decay by emitting one or more photons. For example, $\mathrm{O}^{+7}$ $+\mathrm{H}^{0} \rightarrow \mathrm{O}^{+6 *}+\mathrm{H}^{+1} \rightarrow \mathrm{O}^{+6}+\mathrm{H}^{+1}+$ photons. During the radiative decay, the electron may pass through several excitation levels. The last transition, the $n \geq 2$ to $n=1$ transition, will produce an X-ray photon. For example, in $\mathrm{O}^{+6}$, each $n=2$ to $n=1$ transition produces a $\sim 570$ eV X-ray photon.

The charge transfer interactions, called Solar Wind Charge Exchange (SWCX), are expected to occur between solar wind ions and neutral interstellar atoms that have drifted into the heliosphere (called heliospheric SWCX), and between solar wind ions and material in the Earth's upper atmosphere (called geocoronal SWCX). SWCX interactions with material flowing into the heliosphere were found to outnumber SWCX interactions with material in the Earth's atmosphere (20). The solar wind is non-isotropic and time varying on long and short time scales. The longest timescale is $\sim 11$ years and is associated with the Solar activity cycle. The shortest timescale is on the order of an hour and is caused by Coronal Mass Ejections.

Multiple research groups have modeled the SWCX emission of soft X-ray photons. Concentrating on the $1 / 4 \mathrm{keV}$ X-ray band, and accounting for the state of the Solar Cycle and the sight line geometries during the ROSAT All Sky Survey, (20) calculated the heliospheric and geocoronal SWCX contributions to the $1 / 4 \mathrm{keV}$ flux observed by ROSAT. Their SWCX emission map (Figures 9 and 10 in their paper) is markedly non-isotropic, with two dim 
regions centered on $\ell \sim 220^{\circ}, b \sim-45^{\circ}$ and $\ell \sim 130^{\circ}, b \sim 0^{\circ}$ and with an extended bright region whose intensity peaks near $\ell=240^{\circ}, b=45^{\circ}$. Their conservatively estimated intensity of $1 / 4 \mathrm{keV}$ emission accounts for about half of the diffuse $1 / 4 \mathrm{keV}$ X-ray intensity seen in the Galactic plane and thus accounts for about half of the intensity previously attributed to the Local Bubble in the Galactic plane. The comparison at higher latitudes is more complicated due to the Galactic halo contribution. Subsequently, (21) modeled the heliospheric $\mathrm{O}$ VII SWCX emission at $\sim 570 \mathrm{eV}$ and estimated that as many as 0.8 to 3.4 photons $\mathrm{cm}^{-2}$ $\mathrm{s}^{-1} \mathrm{sr}^{-1}$ could result from SWCX in the slowly varying solar wind, depending on the stage of the Solar cycle and the viewing angle. This range is of the order of what is expected from the Local Bubble. Note that the O VII triplet at $\sim 570 \mathrm{eV}$ lies outside ROSAT's $1 / 4 \mathrm{keV}$ band, but within ROSAT's $3 / 4 \mathrm{keV}$ band, as well as XMM, Chandra, and Suzaku's bandpasses. The Local Bubble is much dimmer in the $3 / 4 \mathrm{keV}$ band than the $1 / 4 \mathrm{keV}$ band. Therefore the LB's O VII spectral features are not expected to be bright and the SWCX photons may account for a relatively larger fraction of the observed intensity.

In addition to the heliospheric and geocoronal SWCX components, there seems to be an even brighter component which may be associated with Coronal Mass Ejections (CMEs). Large CMEs discharge $\sim 10^{11}$ to $\sim 10^{13} \mathrm{~kg}$ of ionized material non-isotropically into the heliosphere over periods of hours. As this material flows outwards through the heliosphere, it is subject to charge exchange that leads to X-ray emission. This is the conclusion drawn by (22) and used to explain the relatively high O VII intensities seen in Chandra and XMM shadowing observations. In one example, $X M M$ observed a pair of shadowing sight lines in 2002, during the solar maximum when CMEs are most common. Suzaku observed the sightlines in 2006, during Solar minimum when CMEs are least common. Between the 2002 and 2006 observations, the "locally produced" O VII intensity dropped from $6.1_{-3.0}^{+2.8}$ to $0.4 \pm 1.1$ photons $\mathrm{cm}^{-2} \mathrm{~s}^{-1} \mathrm{sr}^{-1}(23)$. Not only does a CME provide enough material to explain the large intensity difference, but, as (23) point out, it also provides an explanation for why there was no anomaly in the solar wind proton data recorded by ACE SWEPAM near the time of the $X M M$ observation. ACE monitors the solar wind $\sim 0.01$ AU upstream from the Earth, and it would not have noticed a CME that did not pass through the satellite.

\subsection{Solar Wind Charge Exchange O VI}

Although SWCX is an important contaminant to the observations of diffuse X-rays, it is not a significant contaminant to the $\mathrm{O}$ VI observations. This can be verified by the following estimates. In the first estimate, the O VI column density, $N_{O V I}$, is found from $\int n_{O V I} d l$, where the volume density of $\mathrm{O}$ VI ions, $n_{O V I}$, can be estimated from the volume density of solar wind protons, $n_{p}$, the oxygen-to-hydrogen ratio, $[O / H]$, and the fraction of solar wind oxygen atoms in the $\mathrm{O}^{+5}$ ionization state. The solar wind proton density decreases with distance from the Sun, $r$, as $n_{p}=n_{p 1}\left(r_{1} / r\right)^{2}$, where $r_{1}$ is $1 \mathrm{AU}$ and $n_{p 1}$ is the proton density at $r_{1}$. The proton density at $r_{1}$ can be found from the relationship between the proton density, proton flux, and solar wind velocity (i.e. $n=F / v$ ), using (24)'s estimates for the solar wind proton flux at $r_{1},\left(F_{1}=2 \times 10^{8} \mathrm{~cm}^{-2} \mathrm{~s}^{-1}\right)$, and solar wind velocity (fast wind: $810 \mathrm{~km}$ $\mathrm{s}^{-1}$, slow wind: $442 \mathrm{~km} \mathrm{~s}^{-1}$, simple average: $626 \mathrm{~km} \mathrm{~s}^{-1}$ ). Thus $n_{p 1}=3.2 \mathrm{~cm}^{-3}$. (24)'s estimate for the oxygen-to-hydrogen ratio in the solar wind is also used. The ratio varies from $1 / 1780$ for the slow wind to $1 / 1550$ for the fast wind. The simple average of these numbers yields $[O / H] \sim 6.0 \times 10^{-4}$ oxygens per hydrogen. All of the solar wind oxygen atoms are ionized at the $\mathrm{O}^{+6}$ level or above when they leave the Sun (24). Some of these ions will charge exchange to the $\mathrm{O}^{+5}$ level while in the Solar System, so the upper limit on 
the $\mathrm{O}^{+5}$ to oxygen ratio along a given sightline is 1.0. As a result, the upper limit on $N_{O V I}$ is $1.9 \times 10^{-3} \mathrm{~cm}^{-3} \int\left(r_{1} / r\right)^{2} d l$. The integral is equal to $0.99 \mathrm{AU}$, for the simplest path, which begins at the Earth and proceeds directly away from the Sun to the heliopause located about 100 AU downstream. Thus, the estimated $N_{O V I} \lesssim 2.9 \times 10^{10} \mathrm{~cm}^{2}$. This value is much smaller than the error bars on some of the smallest column densities observed by FUSE, such as $N_{O V I}=2.4 \pm 1.4 \times 10^{12} \mathrm{~cm}^{-2}(9)$, and $N_{O V I}=0.3 \pm 2.3 \times 10^{12} \mathrm{~cm}^{-2}(8)$ ).

The intensity of SWCX-induced O VI resonance line photons $(1032,1038 \AA)$ has not been estimated by $(20 ; 21 ; 22)$. However, an estimate can be made from the information in (21). They predicted the intensity of heliospheric SWCX 72 and $82 \mathrm{eV}$ photons, which are produced by $\mathrm{O}^{+5}$ ions undergoing transitions from the $n=3$ shell to the $n=2$ shell. Note that some of these transitions will place the electron into the $p$ subshell, while others will place it into the $s$ subshell. Those left in the $p$ subshell will then undergo the $2 p^{2} P_{3 / 2}$ to $2 s^{2} S_{1 / 2}$ or the $2 p^{2} P_{1 / 2}$ to $2 s^{2} S_{1 / 2}$ transitions that yield 1032 and $1038 \AA \mathrm{O}$ VI resonance line photons. Thus, the upper limit on the $\mathrm{O}$ VI resonance line intensity due to heliospheric SWCX is set by (21)'s predictions for the 72 and $82 \mathrm{eV}$ photons. The greatest intensity of these photons shown in their Figure 1 maps is $3.3 \times 10^{-9} \mathrm{ergs} \mathrm{s}^{-1} \mathrm{~cm}^{-2} \mathrm{sr}^{-1}$. Thus, the $\mathrm{O}$ VI resonance line intensity can be estimated as $<26$ photons s $\mathrm{s}^{-1} \mathrm{~cm}^{-2} \mathrm{sr}^{-1}$. Even if this upper limit were to be elevated by a factor of several due to a CME along the line of sight, it would be much smaller than the $1 \sigma$ error bars on the FUSE observations of the local $\mathrm{O}$ VI resonance line intensity $\left(\sim 200\right.$ photons s $\mathrm{s}^{-1} \mathrm{~cm}^{-2} \mathrm{sr}^{-1}$ for each of the resonance lines, (18)), showing that that SWCX is not an important factor when evaluating O VI emission observations.

\section{Is There a Hot Local Bubble After All ?}

There is a hot Local Bubble. (25) found that maps of the 1/4 keV X-ray sky are still bright, even after the SWCX intensity is subtracted. Especially noteworthy is that there are regions in the Galactic plane with non-zero net intensity (countrate $\sim 200 \times 10^{6}$ counts s ${ }^{-1}$ $\operatorname{arcmin}^{-2}$ ). Due to the high opacity of the Galactic disk, only soft X-rays that are made locally and a small fraction of the X-rays that come from very bright non-local sources will be observed at Earth. Thus, low latitudes X-rays are almost entirely attributable to the local region and the mapped intensity of low latitude X-rays remaining after SWCX X-rays have been subtracted is almost entirely attributable to the Local Bubble. The story becomes more complicated when one considers the $3 / 4 \mathrm{keV}$ band, or one of its most prominent features, the O VII triplet. According to (22), it is possible that SWCX might account for as much as $100 \%$ of the measured local O VII intensity. However, given the inherent uncertainty in the SWCX O VII estimates, there is room for both the Local Bubble and Solar Wind Charge Exchange. Also, even if the majority of local O VII photons resulted from SWCX events, the Local Bubble would not be doomed. The Local Bubble is not thought to be especially bright in $3 / 4 \mathrm{keV} \mathrm{X}$-rays (the local $3 / 4 \mathrm{keV}$ countrate is only $\sim 1 / 15$ as bright as the local $1 / 4 \mathrm{keV}$ countrate (10)), which makes it easier for a contaminant such as SWCX to produce a large fraction of the observed countrate.

One of the arguments for the continued belief in the Local Bubble is that the O VI column density found between the Earth and nearby stars $(26 ; 8 ; 9)$ implies the presence of hot gas and cannot be explained by SWCX. The O VI ion is a tracer of $\sim 3 \times 10^{5} \mathrm{~K}$ gas. Gas of this temperature is thermally unstable; it does not remain at this temperature for long periods unless hotter gas is present to resupply it. Therefore, it is primarily located in transition zones between hotter and cooler gas. Its existence within the local region has been found by 
Copernicus, which observed measureable quantities of $\mathrm{O}$ VI ions on 2 sight lines terminating within $90 \mathrm{pc}$ of the Sun and several more sight lines terminating within $200 \mathrm{pc}$ of the Sun (26), and later by FUSE, which observed O VI ions and on dozens of sight lines with similar distance ranges $(8 ; 9)$. It should be noted that the original FUSE work has been questioned by (27), who have reprocessed the original data using a more recent version of the FUSE data processing pipeline. They found that some of the previously observed O VI absorption features may be artifacts of the older data processing, while other features might be circumstellar features due to the white dwarf targets. Nonetheless, (27) did not contradict all of the earlier FUSE detections. They found interstellar O VI on several close sight lines, including WD0004+330, WD0354-368, WD2000-561, and WD2331-475. Because they found far less O VI within the LB's radius than had $(8 ; 9)$, they concluded that the interstellar O VI only resides near the LB's periphery, and not within the LB as (8) had concluded. The (27) reanalysis does not extend to the Copernicus data. None of the Copernicus targets were white dwarfs; all of the nearby targets were B stars. Furthermore, the orginal work by $(26 ; 28)$ ruled out circumstellar orgins for the $\mathrm{O}$ VI found in the Copernicus data set. As shown in Section 3.2 the observed O VI ions cannot be attributed to the solar wind ions or SWCX, and so the only choice is that they are associated with hot interstellar gas in the local region, i.e. in the Local Bubble.

Another argument for the Local Bubble's existence is that the Local Cavity must be occupied. If there is no Local Bubble to balance the thermal and magnetic pressure of the material outside the Local Cavity, then the material outside the cavity would expand inwards at the sound speed. Similarly, the Local Cloud that surrounds the Solar System and other clouds that are also embedded in the Local Bubble's volume would also expand at the sound speed. Such expansion, with predicted velocities of about $15 \mathrm{~km} \mathrm{~s}^{-1}$, has not been observed (29). It might be argued that it is the total pressure (including magnetic and cosmic ray pressures) rather than the thermal pressure, alone, which governs the dynamics. Therefore, it may be possible for the total pressure in the Local Cavity to balance that in the embedded clouds and the surrounding material if the non-thermal pressure in the Local Cavity is unusually large. In response, one could point out that some researchers find low magnetic field strengths $(B=0.7 \mu \mathrm{G},(30))$ although other researchers find high magnetic field strengths $(B=8 \mu \mathrm{G},(31))$ on lines of sight terminating within $200 \mathrm{pc}$ of the Sun.

Also note that during the conference, some participants added additional reasons to this list. Steve Snowden pointed out that there is good agreement between the Wisconsin, HEAO 1, and ROSAT $1 / 4 \mathrm{keV}$ band data (32), yet these surveys were made at different times and with different angles between the target and the Sun. Such agreement is not possible if most of the X-rays derive from a strongly time and look-angle dependent source such as the solar system. Furthermore, the anticorrelation between neutral hydrogen column density and soft X-ray intensity cannot be explained by SWCX. For a debate of the arguments for and against the existence of the Local Bubble, the reader is refered to the session report in this volume (33).

\section{Revising the Local Bubble}

If, as (20) conservatively estimated, half of the local $1 / 4 \mathrm{keV}$ X-rays are due to SWCX and half are due to the hot Local Bubble, then the Local Bubble must be considerably weaker than previously thought. Its electron density and thermal pressure would be reduced by a factor of $1 / \sqrt{2}$ from their previous values to $n_{e} \sim 0.005 \mathrm{~cm}^{-3}$ and $P_{t h} / k \sim 11,000 \mathrm{~K} \mathrm{~cm}^{-3}$, respectively, if the gas temperature and path-length remain unchanged. These reductions are 
comforting, because the previous pressure estimate $\left(P_{t h} / k \sim 15,000 \mathrm{~K} \mathrm{~cm}^{-3},(11)\right)$ was far greater than that of the embedded warm clouds $\left(P_{t h} / k \sim 2000\right.$ to $\left.3000 \mathrm{~K} \mathrm{~cm}^{-3}\right)$.

The reductions may also help to explain the dearth of Fe IX, Fe X, and Fe XI $\sim 72 \mathrm{eV}$ photons observed by the X-ray calorimeter (34) and CHIPS (35). This is a muddy issue, because $C H I P S$ observed a significantly lower intensity than did the calorimeter and because preferential depletion of gas phase iron relative to other elements can partly explain the dearth of photons observed by CHIPS. Nonetheless, if SWCX caused half of the observed local $1 / 4 \mathrm{keV}$ emission but did not contribute Fe IX, Fe X, or Fe XI photons, then the LB's emission measure would be half that previously expected (thus half of $0.0038 \mathrm{pc}^{-1} \mathrm{~cm}^{-6}$ expected for the CHIPS observations assuming a temperature of $10^{6.1} \mathrm{~K}$, (35)) bringing it well within the $95 \%$ upper limit contour found from their observations $\left(0.0036 \mathrm{pc}^{-1} \mathrm{~cm}^{-6}\right)$.

A weaker Local Bubble requires $1 / \sqrt{2}$ less initial input energy. Rather than a few supernova explosions, perhaps only one or two would be sufficient for evacuating and heating the bubble. Another consequence of considering a significant SWCX contribution is the possibility that it has skewed the LB temperature measurements. The present observational evidence tells us the shape of the combined LB+SWCX spectrum. The SWCX spectrum must be subtracted in order to find the true shape of the LB spectrum. Therefore, the Local Bubble could be hotter or cooler than previously believed. There may already be observational hints of this effect. For example, XMM and Suzaku observed the same cloud shadow 4 years apart. The $X M M$ data was later found to be contaminated by a SWCX CME. Before that was known, the XMM data, in combination with ROSAT $1 / 4 \mathrm{keV}$ data, was used to find the temperature of the local plasma (i.e. the temperature of the SWCX and LB spectra, combined; $\log (T)=6.06_{-0.04}^{+0.02} \mathrm{~K},(36)$ ). But, the Suzaku data, which is not known to be contaminated by a CME, in combination with the same ROSAT data, yielded $\log (T)=5.96_{-0.04}^{+0.05} \mathrm{~K},(23)$.

Lastly, as a result of SWCX contamination, some LB models that had previously been found to be in conflict with the observations may need to be reevaluated. For example, the break-out model (in which the Local Bubble gas was heated and then expanded so fast that the ions adiabatically cooled faster than they recombined, $(16)$ ) had been eliminated on the grounds that its ratios of $1 / 4 \mathrm{keV}$ to $\mathrm{O}$ VI emission, $\mathrm{C}$ III emission, and $\mathrm{O}$ VI absorption column density were higher than the observations indicated. But, if the LB's soft X-ray intensity were to be revised downwards significantly, then the break-out model would require reconsideration.

\section{How We Can Make Progress}

Continued work on SWCX estimates and the uncertainties on those estimates will enable continued progress in determining how best to apportion the observed local X-ray intensity between the SWCX and LB components. Continued work on estimating the SWCX 1/4 to $3 / 4 \mathrm{keV}$ spectrum and its subtraction from the observationally derived local spectrum will enable revised estimates of the LB's spectrum and hence the plasma's temperature. But, such a statement must be qualified, because 1.) some estimates already exist (19) although the spectral subtraction has yet to be done; and 2.) if the SWXC contribution is large, then the uncertainty in the LB spectrum will be very large.

Clever strategies are needed in order to set observational constraints on the SWCX intensity. Perhaps these strategies will use the temporal and spatial variations in the model SWCX emission. Or perhaps, they will use a shadowing strategy to observe the SWCX spectrum without "contamination" by Galactic X-rays. Observations in the $1 / 4 \mathrm{keV}$ band would be very useful, although they may not be attainable with current instruments. Concentrating on 
this band is necessary because the observed local X-ray spectrum peaks in the $1 / 4 \mathrm{keV}$ band, and as a result, observations in higher bands alone are not sufficient for constraining fits to spectral models. In the future, very high spectral resolution observations will be useful. Few to several-eV resolution is necessary for unambiguous measurements of atomic transitions in the cascade from high $n$ levels. Such measurements will include the determination of the ratio of intensities in the O VII "triplet" at 561, 569, and $574 \mathrm{eV}$ and measurements of the weak O VIII Ly $\delta$ line at $836 \mathrm{eV}$ and the weak C VI Ly $\gamma$ line at $459 \mathrm{eV}$ predicted by (37).

Acknowledgements I would like to thank all of the members of my splinter group for their lively and informative contributions to the discussions of the Local Bubble and to thank the conference organizers for enabling these exchanges. I would also like to acknowledge helpful comments on the manuscript from Jeff Linsky, informative conversations with Dave Henley, an excellent explanation of solar wind charge exchange by Tom Cravens, and funding through the NASA LTSA grant NNG04GD78G.

\section{References}

1. McCammon, D. \& Sanders, W. T., ARA\&A, 28, 657-658 (1990)

2. Burrows, D. N. \& Mendenhall, J. A., Nature, 351, 629-631 (1991)

3. Snowden, S. L., Mebold, U., Hirth, W., Herbstmeier, U., \& Schmitt, J. H. M., Science, 252, 1529-1532 (1991)

4. Bunner, A. N., Coleman, P. L., Kraushaar, W. L., McCammon, D., \& Williamson, F. O., ApJ, 179, 781-788 (1973)

5. Lisse, C. M., Dennerl, K., Englhauser, J., Harden, M., Marshall, F. E., Mumma, M. J., Petre, R. Pye, J. P., Ricketts, M. J., Schmitt, J., Trümper, J., \& West, R. G., Science, 274, 205 - 209 (1996)

6. Kuntz, K. D., \& Snowden, S. L., ApJ, 543, 195-215 (2000)

7. Shelton, R. L., \& Cox, D. P., ApJ, 434, 599-613 (1994)

8. Oegerle, W. R., Jenkins, E. B., Shelton, R. L., Bowen, D. V., \& Chayer, P., ApJ, 622, 377-389 (2005)

9. Savage, B. D. \& Lehner, N. ApJS, 162, 134-160 (2006)

10. Snowden, S. L., McCammon, D., \& Verter, F. ApJ, 409, L21-L24 (1993)

11. Snowden, S. L., Egger, R., Finkbeiner, D. P., Freyberg, M. J., \& Plucinsky, P. P., ApJ, 493, 715-729 (1998)

12. Lallement, R., Welsh, B. Y., Vergely, J. L., Crifo, F., \& Sfeir, D., A\&A, 411, 447-464 (2003)

13. Cox, D. P., \& Anderson, P. R., ApJ, 253, 268-289 (1982)

14. Edgar, R. J., ApJ, 308, 389-400 (1986)

15. de Avillez, M. A., \& Breitschwerdt, D., Rev. Mex. AA Ser. Conf., 15, 299-299 (2003)

16. Breitschwerdt, D., \& Schmutzler, T., Nature, 371, 774-777 (1994)

17. Welsh, B. Y., Sallmen, S., Sfeir, D., Shelton, R. L., \& Lallement, R., A\&A, 394, 691-699 (2002)

18. Shelton, R. L., ApJ, 589, 261-269 (2003)

19. Cravens, T. E., ApJ, 532, L153-156 (2000)

20. Robertson, I. P. \& Cravens, T. E., JGR, 108(A10), 8031, LIS6-1 - 6-10 (2003)

21. Koutroumpa, D., Lallement, R., Kharchenko, V., Dalgarno, A., Pepino, R., Izmodenov, V., \& Quémerais, E., A\&A, 460, 289-300 (2006)

22. Koutroumpa, D., Acero, F., Lallement, R., Ballet, J., \& Kharchenko, V., A\&A, 475, 901-914 (2007) 
23. Henley, D. B. \& Shelton, R. L., ApJ, 676, 335-350 (2008)

24. Schwadron, N. A. \& Cravens, T. E., ApJ, 544, 558 (2000)

25. Robertson, I. P. Cravens, T. E., \& Snowden, S. L., American Geophysical Union, 2003, SH11C1126 (2003)

26. Jenkins, E. B., ApJ, 219, 845-860 (1978)

27. Barstow, M. A., Boyce, D. D., Barstow, J. K., Forbes, A. E., Welsh, B. Y., \& Lallement, R., Astrophysics \& Space Science, submitted (2008)

28. Jenkins, E. B., ApJ, 220, 107-123 (1978)

29. Slavin, J. D., personal communication (2007)

30. Spangler, S., personal communication (2007)

31. Andersson, B.-G., \& Potter, S. B., ApJ, 640, L51-L54 (2006)

32. Snowden, S. L., Freyberg, M. J., Plucinsky, P. P., Schmitt, J. H. M. M., Trümper, J., Voges, W., Edgar, R. J., McCammon, D., \& Sanders, W. T., ApJ, 454, 643-653 (1995)

33. Shelton, R. L., Space Sciences Reviews, this volume, submitted (2008)

34. McCammon, D., et al., ApJ, 576, 188-203 (2002)

35. Hurwitz, M., Sasseen, T. P., \& Sirk, M. M., ApJ, 623, 911-916 (2005)

36. Henley, D. B., Shelton, R. L., \& Kuntz, K. D., ApJ, 661, 304-319, (2007)

37. Wargelin, B. J., Beiersdorfer, P., \& Brown, G. V., CaJPh, 86, 151-169 (2008) 